\title{
Cardiac magnetic resonance imaging of myocarditis and pericarditis following COVID-19 vaccination: a multicenter collection of 27 cases
}

\author{
Emanuele Angelo Di Dedda ${ }^{1,2} \cdot$ Andrea Barison $^{3}$ - Giovanni Donato Aquaro ${ }^{3}$. Tevfik F Ismail ${ }^{4}$ Alina Hua $^{4}$. \\ Cesare Mantini ${ }^{5}$. Fabrizio Ricci ${ }^{5}$. Gianluca Pontone ${ }^{6}$ - Alessandra Volpe ${ }^{7,8}$ - Francesco Secchi ${ }^{9,10}$ - Paolo Di Renzi ${ }^{11}$. \\ Luigi Lovato $^{12}$. Fabio Niro ${ }^{12}$. Carlo Liguori ${ }^{13}$. Chiara De Biase ${ }^{14} \cdot$ Lorenzo Monti $^{2}$. Antonio Cirò ${ }^{15}$. \\ Riccardo Marano ${ }^{16,17}$. Luigi Natale ${ }^{16,17}$. Eleonora Moliterno ${ }^{17}$. Antonio Esposito ${ }^{18,19}$. Davide Vignale ${ }^{18,19}$. \\ Riccardo Faletti ${ }^{20}$ - Marco Gatti ${ }^{20}$ - Michele Porcu ${ }^{21}$ - Luca Saba ${ }^{21}$ - Cristina Chimenti ${ }^{22} \cdot$ Nicola Galea $^{23}$. \\ Marco Francone ${ }^{1,2}$ (D)
}

Received: 27 August 2021 / Revised: 11 December 2021 / Accepted: 4 January 2022 / Published online: 1 March 2022

(C) The Author(s), under exclusive licence to European Society of Radiology 2022

\begin{abstract}
Objectives To assess clinical and cardiac magnetic resonance (CMR) imaging features of patients with peri-myocarditis following Coronavirus Disease 2019 (COVID-19) vaccination.

Methods We retrospectively collected a case series of 27 patients who underwent CMR in the clinical suspect of heart inflammation following COVID-19 vaccination, from 16 large tertiary centers. Our patient's cohort was relatively young (36.6 \pm 16.8 years), predominately included males $(n=25 / 27)$ with few comorbidities and covered a catchment area of approximately 8 million vaccinated patients.

Results CMR revealed typical mid-subepicardial non-ischemic late gadolinium enhancement (LGE) in 23 cases and matched positively with CMR T2 criteria of myocarditis. In 7 cases, typical hallmarks of acute pericarditis were present. Short-term follow-up (median = 20 days) from presentation was uneventful for 25/27 patients and unavailable in two cases.

Conclusions While establishing a causal relationship between peri-myocardial inflammation and vaccine administration can be challenging, our clinical experience suggests that CMR should be performed for diagnosis confirmation and to drive clinical decision-making and follow-up.

Key Points

- Acute onset of dyspnea, palpitations, or acute and persisting chest pain after COVID-19 vaccination should raise the suspicion of possible myocarditis or pericarditis, and patients should seek immediate medical attention and treatment to help recovery and avoid complications.

- In case of elevated troponin levels and/or relevant ECG changes, cardiac magnetic resonance should be considered as the best noninvasive diagnostic option to confirm the diagnosis of myocarditis or pericarditis and to drive clinical decision-making and follow-up.
\end{abstract}

Keywords Magnetic resonance imaging $\cdot$ COVID-19 $\cdot$ Vaccination $\cdot$ Myocarditis $\cdot$ Pericarditis

Position statement on COVID-19 vaccines: The authors are firm supporters of the COVID-19 vaccination campaign and vaccinated themselves as well.

Marco Francone marco.francone@hunimed.eu

$\begin{array}{ll}\text { Abbreviations } & \\ \text { AHA } & \text { American Heart Association } \\ \text { CDC } & \begin{array}{l}\text { Centers for Disease } \\ \text { Control and Prevention }\end{array} \\ \text { Cine-SSFP } & \text { Cine steady-state free precession } \\ \text { CMR } & \text { Cardiac magnetic resonance } \\ \text { CMRI } & \text { Cardiac magnetic resonance imaging } \\ \text { COVID-19 } & \text { Coronavirus disease 2019 } \\ \text { ECG } & \text { Electrocardiogram } \\ \text { ECV_cmr } & \text { Myocardial extracellular volume } \\ & \text { fraction estimated by CMR }\end{array}$




\begin{tabular}{|c|c|}
\hline EF & Ejection fraction \\
\hline FU days & Follow-up days from presentation \\
\hline hs-cTnI & High-sensitivity cardiac troponin I \\
\hline hs-cTnT & High-sensitivity cardiac troponin $\mathrm{T}$ \\
\hline LGE & Late gadolinium enhancement \\
\hline $\begin{array}{l}\text { LGE segments } \\
\text { (AHA) }\end{array}$ & $\begin{array}{l}\text { LGE left ventricular distribution based on } \\
\text { the " } 17 \text { segments cardiac segmentation } \\
\text { model" by the American }\end{array}$ \\
\hline & Heart Association \\
\hline LV & Left ventricular \\
\hline LVEDVI & $\begin{array}{l}\text { Indexed left ventricular } \\
\text { end-diastolic volume }\end{array}$ \\
\hline LVEDVI_cmr & LVEDVI estimated by CMR \\
\hline LVEF & Left ventricular ejection fraction \\
\hline LVEF_cmr & LVEF estimated by CMR \\
\hline mRNA & Messenger ribonucleic acid \\
\hline n.v. & Normal values \\
\hline Pericarditis $\mathrm{cmr}$ & Pericarditis detected by CMR \\
\hline ROI & Region of interest \\
\hline SARS-CoV-2 & $\begin{array}{l}\text { Severe acute respiratory syndrome corona- } \\
\text { virus } 2\end{array}$ \\
\hline T2w-STIR & T2-weighted short-tau inversion recovery \\
\hline VAERS & Vaccine Adverse Event Reporting System \\
\hline
\end{tabular}

Since the beginning of the global severe acute respiratory syndrome coronavirus 2 (SARS-CoV-2) pandemic, an unprecedented massive effort has been carried out worldwide to rapidly provide acquired immunity against the development of the coronavirus disease 2019 (COVID19) $[1]$.

As of December 2021, over 8.2 billion doses of a range of different COVID-19 vaccines have been administered, prioritizing distribution to categories that are at highest risk of complications and/or transmission, such as the elderly and the healthcare workers.

While reported side effects following these vaccines have been mild and short-lasting in the overwhelming majority of cases, some series of rare but more significant complications have been collected in various international registries and databases [2].

Myocardial and/or pericardial inflammation is a rare yet known adverse event that has been described in relation to several vaccines (from influenza to smallpox) and also, in recent reports, following SARS-CoV-2 vaccine administration $[3,4]$.

In the USA, as of November 10, 2021, the Vaccine Adverse Event Reporting System (VAERS) has received 1793 reports of myocarditis or pericarditis happening after COVID-19 vaccination [2]. Of these, the Centers for Disease Control and Prevention (CDC) and the Food and Drug Administration (FDA) did confirm 1049 reports of myocarditis or pericarditis, particularly among male adolescents and young adults aged below 30 after messenger ribonucleic acid (mRNA) COVID-19 vaccination [2].

The underlying pathogenesis is reasonably considered to be multifactorial and likely dependent on the activation of an uncontrolled autoimmune response to the vaccine triggered by molecular mimicry and cross-reaction mechanisms occurring in genetically susceptible individuals [4].

While establishing a causal relationship between myocardial and/or pericardial inflammation and vaccine administration can be challenging, recognition of such a clinical entity can be relevant, not only for epidemiological purposes but also to define the appropriate clinical management and follow-up.

The diagnostic contribution of cardiac magnetic resonance (CMR) to non-invasively depict COVID-19-associated myocarditis and pericarditis has been already extensively described in the acute/active and chronic setting of the disease [5].

We retrospectively collected data from a series of 23 cases observed by 16 large tertiary centers in the period from March to July 2021, representing patients in which CMR was performed between 1 and 25 days after vaccination in the clinical setting of a suspected cardiac involvement. Four patients were scanned between 32 and 82 days after vaccination, due to clinical relapse of a previously documented acute myocarditis.

Diagnosis of acute myocarditis was established according to the updated Lake-Louise criteria [6].

Detailed clinical and imaging features of our patient cohort, composed of a total of 27 patients, are summarized in Table 1.

Briefly, our patient population was relatively young (average age $36.6 \pm 16.8$ years), mostly included males $(n=25 / 27)$ and with few comorbidities; notably, autoimmune disorders were observed in $3 / 27$ cases. In addition to suspected post-vaccine forms of myocardial injury, all recruiting centers were also asked to collect data for all patients who received a CMR diagnosis of acute peri-myocarditis in the same observational period, for comparative purposes. With this regard, our consortium has observed overall 238 cases of myocarditis, including 27 cases in vaccinated patients and 211 in unvaccinated individuals $(n=14$ cases with history of COVID-19 disease ; $n=197$ unvaccinated without history of COVID-19 disease); a descriptive summary of patients' risk factors and comorbidities among these different groups is displayed in Table 2.

In vaccinated patients, CMR diagnosis of myocarditis and/ or pericarditis more commonly followed immunization with mRNA vaccines $(n=24 / 27)$, after the second jab $(n=15 / 27)$, and within 10 days from administration $(n=22 / 27$; average 8 \pm 9 days). Clinical presentations included chest pain $(n=25 /$ $27)$, palpitations $(n=10 / 27)$, arthralgias and myalgias $(n=$ $9 / 27)$, and dyspnea $(n=7 / 27)$. High-sensitivity cardiac troponin $\mathrm{T}$ (hs-cTnT) or high-sensitivity cardiac troponin I (hscTnI) levels were systematically elevated in 27/27 cases and associated with a variable spectrum of electrocardiogram 
4354

Eur Radiol (2022) 32:4352-4360

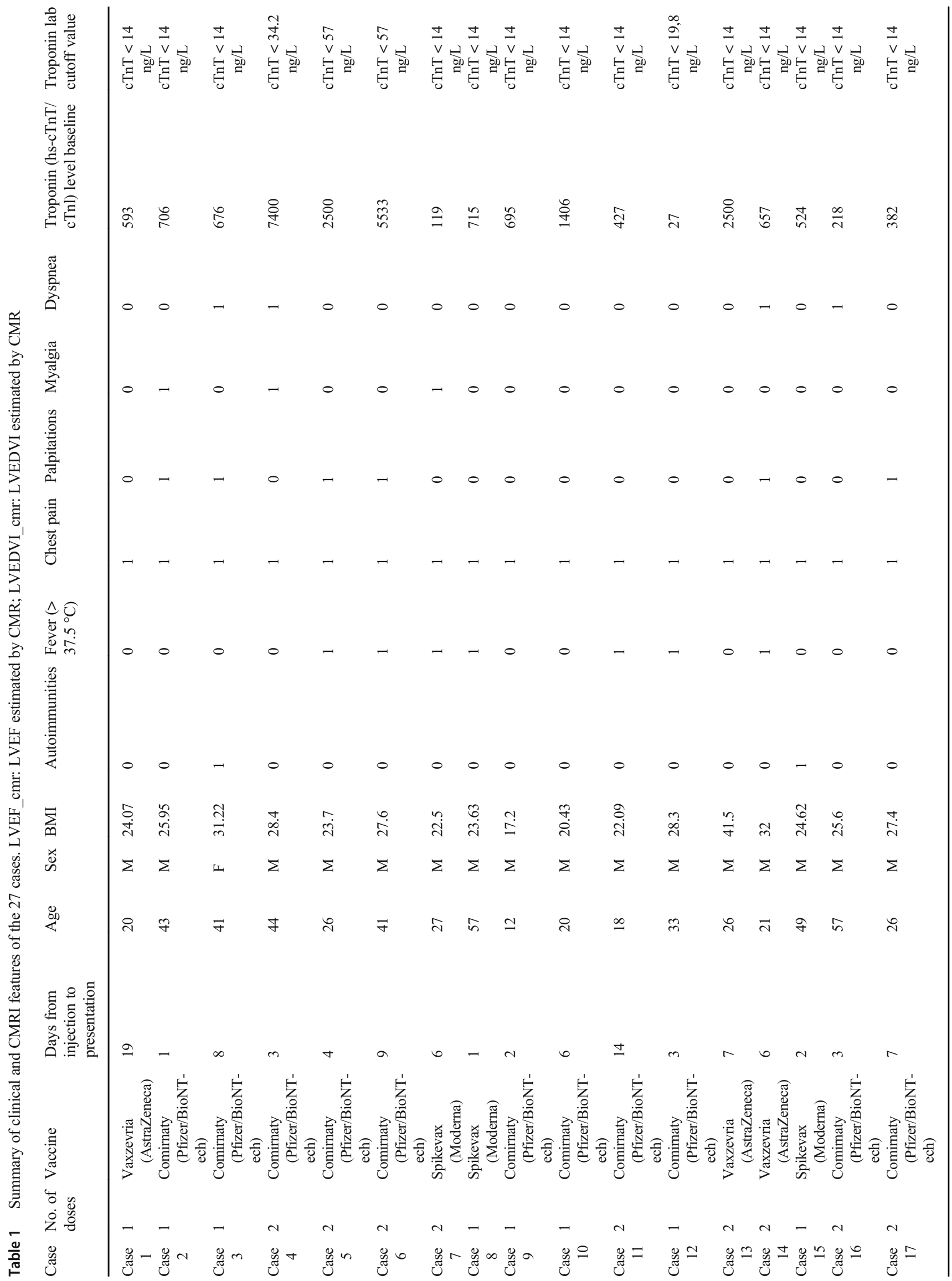

Springer 


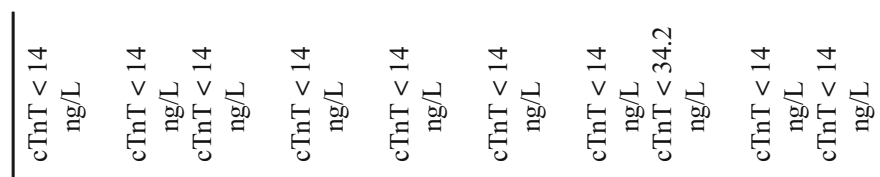

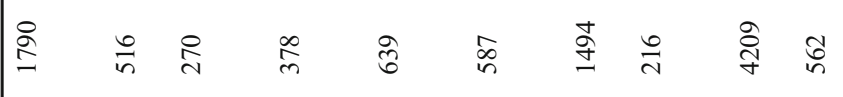

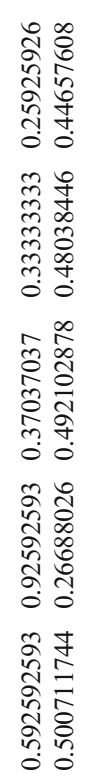

छ赵

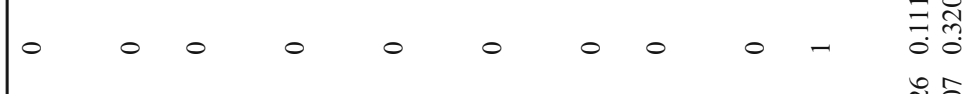

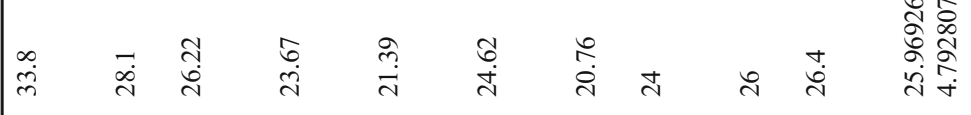

$\Sigma \sum \Sigma \Sigma \Sigma \Sigma \Sigma \Sigma \Sigma \Sigma \Sigma$

i⿱⺈

in ते मे ल ले ब ते

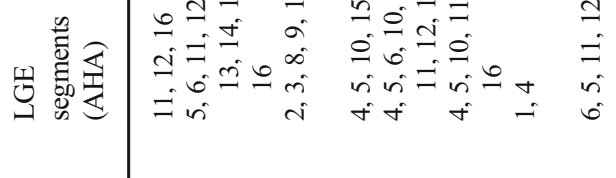

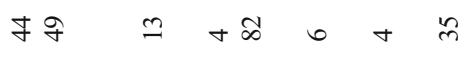

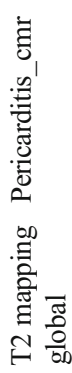

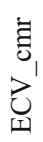

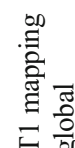

00

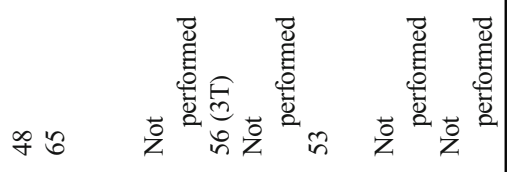

严

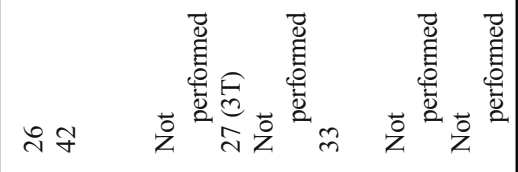

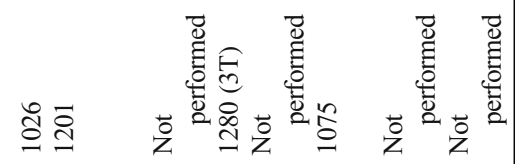

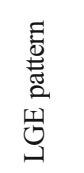

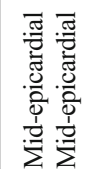

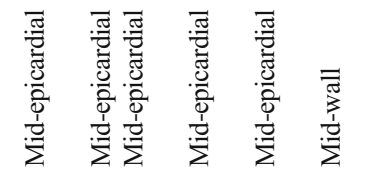

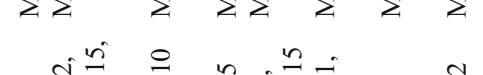

界

है

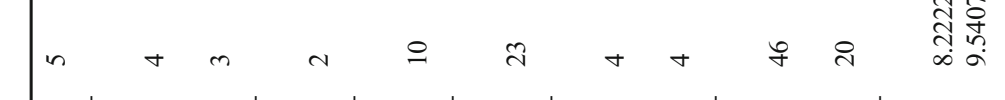

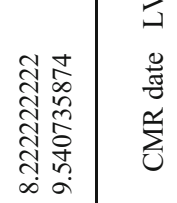

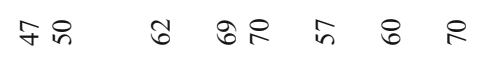

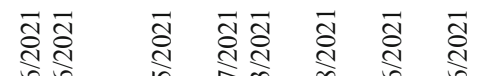

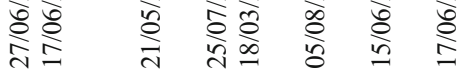

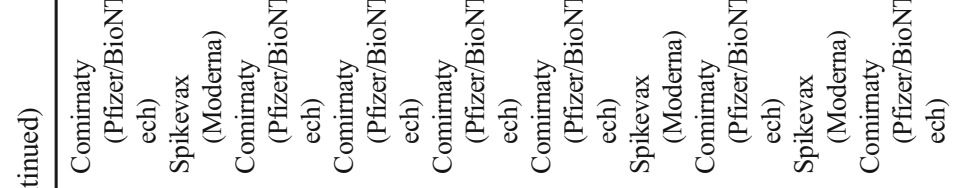

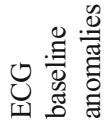

总

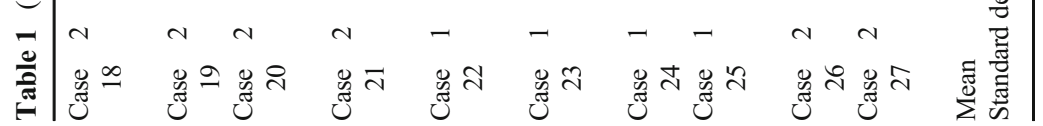

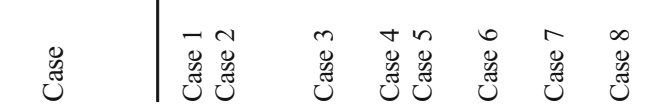




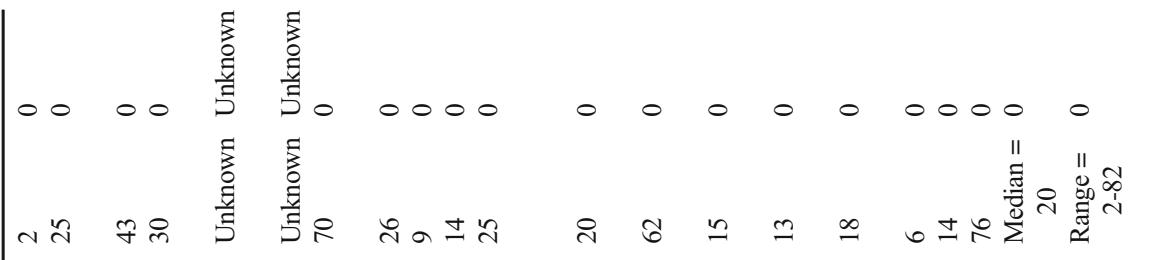

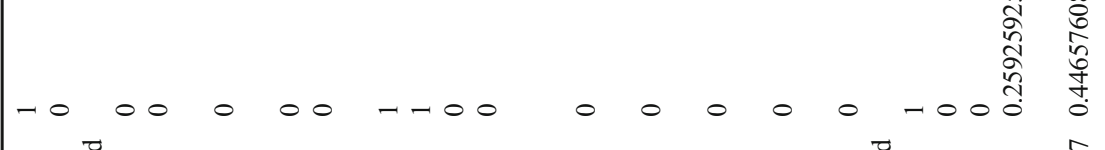

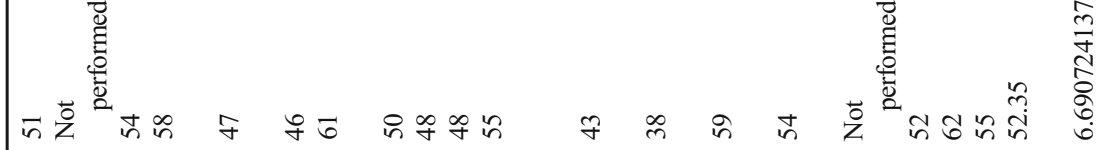

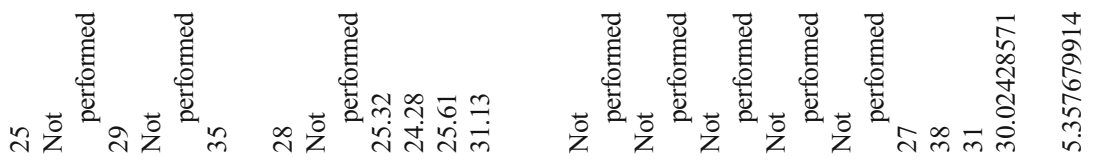

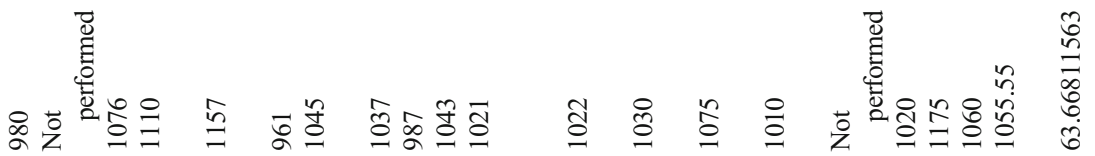

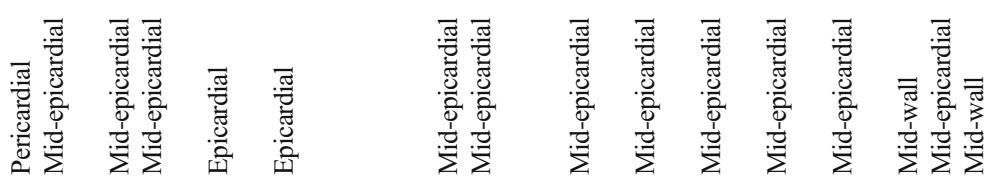

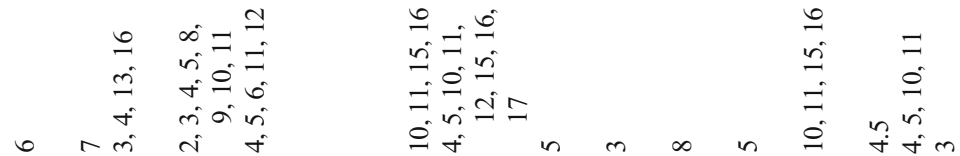

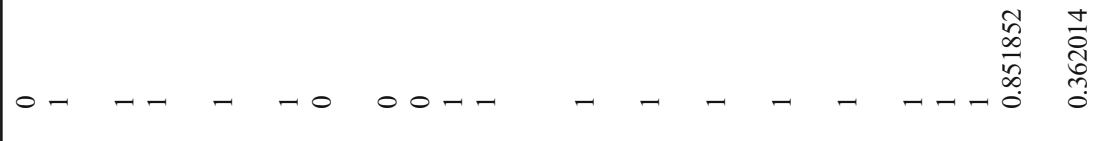

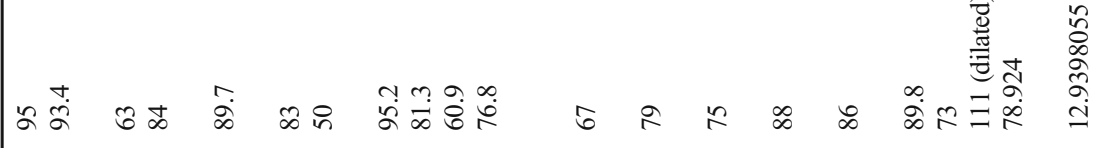

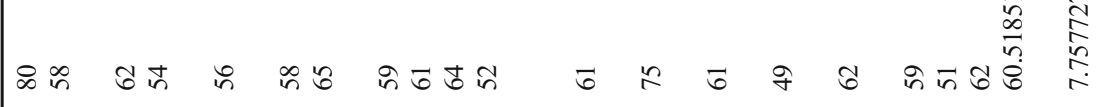

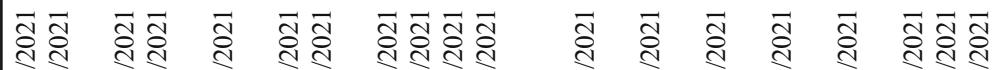

$$
\begin{aligned}
& \text { 方令 }
\end{aligned}
$$

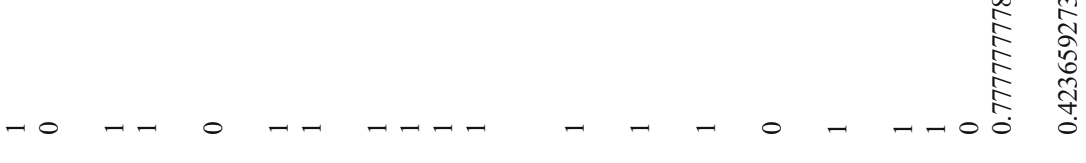

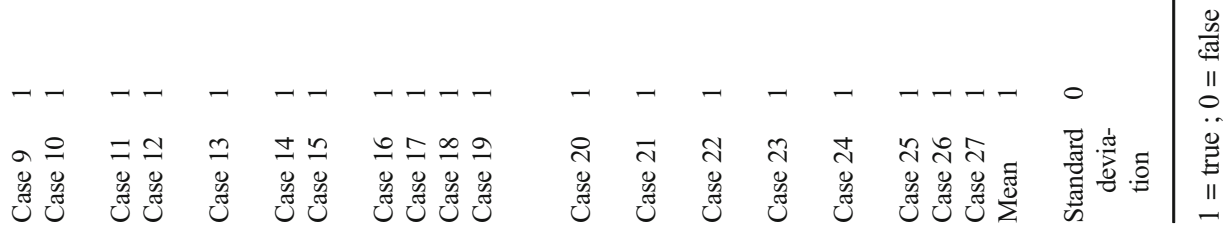


Table 2 Descriptive table reporting prevalence of cardiovascular risk factors and main comorbidities among consecutive patients with a CMR diagnosis of myocarditis and/or pericarditis, observed in the period March-July 2021. Our cohort is categorized into 3 groups: vaccinated: $n=27$ vaccinated patients, COVID-19+ (unvaccinated): $n=14$ unvaccinated patients with diagnosis of acute or healed COVID-19 disease (based on clinical presentation and PCR confirmation), and COVID-19- (unvaccinated): $n=197$ patients, unvaccinated and without history of COVID-19 disease. Definitions of listed risk factors and comorbidities: hypertension $=$ systolic blood pressure $\geq 130 \mathrm{mmHg}$ or a diastolic blood pressure $\geq 80 \mathrm{mmHg}$ or current medical treatment for hypertension; diabetes $=$ fasting glucose $>126 \mathrm{mg} / \mathrm{dL}$ or current treatment; smoking $=$ current smoker or ex-smoker with suspension less than 5 years before observation; hyperlipidemia $=\mathrm{LDL}>130 \mathrm{mg} / \mathrm{dL}$ or current treatment; moderate/high physical activity = at least $150 \mathrm{~min}$ per week of moderate-intensity aerobic activity or $75 \mathrm{~min}$ per week of vigorous aerobic activity, or a combination of both; autoimmunities = history of autoimmune diseases

\begin{tabular}{llllllllll}
\hline & $\begin{array}{l}\text { Age } \\
\text { years, } \\
(\text { mean })\end{array}$ & $\begin{array}{l}\text { Gender } \\
(\% \text { male })\end{array}$ & $\begin{array}{l}\text { BMI }(\mathrm{kg} / \\
\left.\mathrm{m}^{2}\right) \\
(\mathrm{mean})\end{array}$ & $\begin{array}{l}\text { Hypertension } \\
(\%)\end{array}$ & $\begin{array}{l}\text { Diabetes } \\
(\%)\end{array}$ & $\begin{array}{l}\text { Smoking } \\
(\%)\end{array}$ & $\begin{array}{l}\text { Moderate/high } \\
\text { physical activity } \\
(\%)\end{array}$ & $\begin{array}{l}\text { Hyperlipidemia } \\
(\%)\end{array}$ & $\begin{array}{l}\text { Autoimmunities } \\
(\%)\end{array}$ \\
\hline $\begin{array}{l}\text { Vaccinated } \\
\text { COVID-19+ } \\
\quad \text { (unvaccinated) }\end{array}$ & 36.6 & 92.6 & 25.9 & 22.8 & 6.2 & 20.4 & 35.7 & 22.6 & 11.1 \\
$\begin{array}{c}\text { COVID-19- } \\
\text { (unvaccinated) }\end{array}$ & 38.2 & 84.8 & 26.2 & 25.5 & 13 & 29.7 & 22.4 & 44.8 & 9.8 \\
\hline
\end{tabular}

(ECG) abnormalities including ST-segment elevation and Twave inversion ( $n=21 / 27)$.

CMR revealed typical mid-subepicardial non-ischemic late gadolinium enhancement (LGE) in 23 cases and matched positively with CMR T2 criteria of myocarditis (Fig. 1). In 7 cases, CMR showed typical hallmarks of acute pericarditis (effusion with thickening and/or enhancement of pericardial layers).

Left ventricular (LV) systolic function was mildly reduced in $3 / 27$ cases and normal in the remaining population (average ejection fraction: $60.5 \pm 7.7 \%$ ); indexed LV end-diastolic volume (LVEDVI) was normal in all cases $\left(79 \pm 13 \mathrm{~mL} / \mathrm{m}^{2}\right)$, except for an 80-year-old male and a 41-year-old male presenting with a mildly dilated LV cavity (111 and 121 $\mathrm{mL} / \mathrm{m}^{2}$, respectively).

Short-term follow-up from presentation was uneventful for $25 / 27$ patients (median $=20$ days; range $=2-82$ days) and unavailable in two cases.

We collected a case series from the joint efforts of 16 tertiary referral centers, roughly covering a catchment area of approximately 8 million patients vaccinated with at least one dose in the period from March to July. We could therefore estimate an incidence of approximately 3.4 observed cases of myocarditis per million administered doses. Our incidence is significantly lower as compared to most international registries, in which a range of 8.3-34 cases per million was reported (see Fig. 2) [2, 7-9].

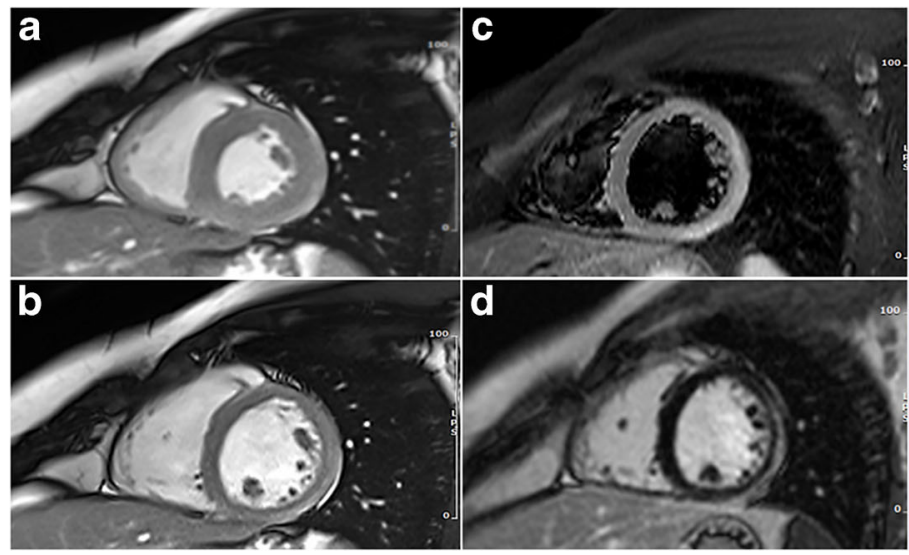

Fig. 1 Acute myocarditis 4 days after Spikevax (Moderna) vaccine administration in a 29-year-old patient (images refer to patient $n .19$ from Table 1) presenting with infarct-like symptoms of acute chest pain, with ECG ST-elevation changes and troponin rise. End-systolic and end-diastolic cine-SSFP frames (a and b) show a non-dilated and functionally preserved left ventricular cavity (EF 61\%; LVEDVI: 76.8 $\mathrm{mL} / \mathrm{m}^{2}$ ). Typical CMR hallmarks of an acute myocarditis can be observed in "edema-weighted" T2w-STIR short axis plane (c), consisting of the presence of a non-ischemic epicardial stria of high

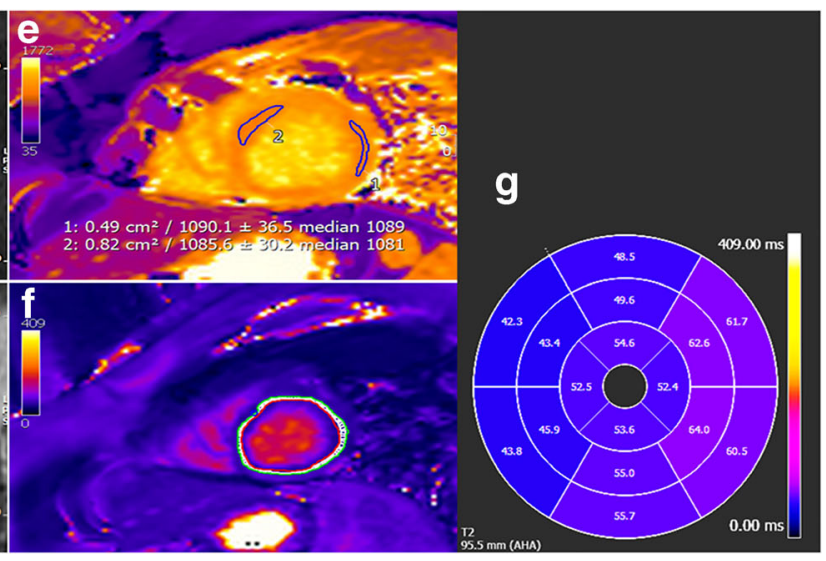

signal intensity involving the anterior- and infero-lateral mid-basal wall (arrows) and closely matching with LGE findings (d) (mid-ventricular level shown). Acute inflammation was also confirmed at myocardial mapping images showing focally increased native T1 mapping (1090 ms of a ROI on the middle-apical lateral wall; n.v. 950-1000 ms; e) and T2 mapping values (avg. $55 \mathrm{~ms}$; n.v. $<50 \mathrm{~ms}$; f) (AHA segments $\mathrm{T} 2$ mapping values shown in $\mathbf{g}$ ). The patient's clinical course was benign and uneventful at 25 days follow-up 


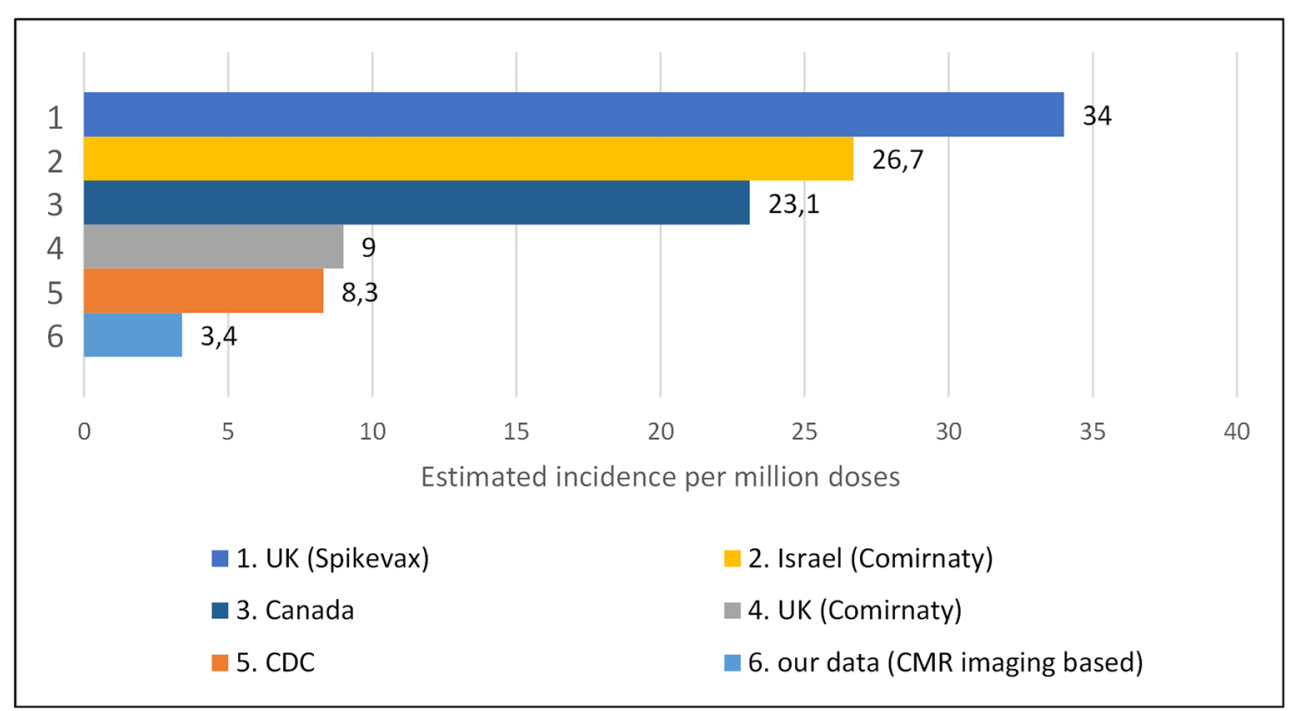

Fig. 2 Estimated incidences of myocarditis after COVID-19 vaccine administration derived from our data and as reported in the following government registries or studies: UK (Spikevax) = reported incidence of myocarditis (34 per million doses) after Spikevax (Moderna) vaccine administration in the UK (government report) [7]; Israel (Comirnaty) = reported incidence of myocarditis (26.7 per million doses) after Comirnaty (Pfizer-BioNTech) vaccine administration in Israel (observational retrospective study based on Ministry of Health database) [8]; Canada $=$ reported incidence of myocarditis $(23.1$ per

This reflects an intrinsic selection difference of our study, in which diagnosis was established with a non-invasive gold standard technique as CMR instead of using clinical diagnostic criteria, like in the Vaccine Adverse Event Reporting System (VAERS), for the CDC, which is a passive reporting system that relies on individuals to send in reports of their experiences [2].

Our findings need to be cautiously contextualized and commented on, because of their potential implications on the perception of vaccine safety by the general population.

A clear causative relationship cannot be established as we only referred to a post-vaccination temporal criterion; moreover, the background prevalence of myocarditis remains uncertain but is likely to be $\sim 22$ per 100,000 [10]. Finally, myocarditis and pericarditis are also both recognised complications of SARS$\mathrm{CoV}-2$ and it is entirely plausible that there are overlapping mechanisms involved in both natural infection and vaccinemediated autoimmunity [11].

Even though we discussed about suspected cardiac side effects of the vaccine, the benefits of the immunization in preventing severe morbidity and mortality from SARS-CoV2 infection still outweigh the risks of complications after vaccine administration [12].

Further work is required to establish whether there are any adverse sequelae associated with the cases of acute myocarditis observed in this case series; however, the largely preserved LV function and pattern of late enhancement may portend a good prognosis, although the presence of LGE highlights the need for careful surveillance. million doses) after COVID-19 vaccine administration in Canada (government report) [9]; UK (Comirnaty) = reported incidence of myocarditis ( 9 per million doses) after Comirnaty (Pfizer-BioNTech) vaccine administration in the UK (government report) [7]; CDC = reported incidence of myocarditis (8.3 per million doses) after COVID19 vaccines administration in the USA (CDC report) [2]; current research $=$ estimated incidence (3.4 per million doses) from CMR data reported in the present study

Acute onset of dyspnea, palpitations, or acute and persisting chest pain after vaccination should raise the suspicion of possible myocarditis or pericarditis, and patients should seek immediate medical attention and treatment to help recovery and avoid complications. In case of elevated troponin levels and/or relevant ECG changes, CMR should be considered as the best non-invasive diagnostic option to confirm the diagnosis and to drive clinical decision-making and follow-up.

Funding The authors declare this study received no funding.

\section{Declarations}

Guarantor The scientific guarantor of this publication is Prof. Marco Francone, $\mathrm{MD}, \mathrm{PhD}$.

IRCCS Humanitas Research Hospital, Department of Biomedical Sciences of Humanitas University

ViaRita Levi Montalcini 4, 20072, Pieve Emanuele, Milan, Italy

E-mail: marco.francone@hunimed.eu; phone: +39 0282243076; fax $+390282242299$

Conflict of interest The authors of this manuscript declare no relationships with any companies whose products or services may be related to the subject matter of the article.

Statistics and Biometry No complex statistical methods were necessary for this paper.

Informed Consent Written informed consent was obtained from all subjects (patients) in this study. 
Ethical Approval Ethical approval was obtained from IRB on 25th May 2021, number 2551.

\section{Methodology}

- retrospective

- observational

- multicenter study

\section{References}

1. Wu Z, McGoogan JM (2020) Characteristics of and important lessons from the coronavirus disease 2019 (COVID-19) outbreak in China: summary of a report of 72314 cases from the Chinese Center for Disease Control and Prevention. JAMA. 323:1239-1242

2. Centers for Disease Control and Prevention (2021) Selected adverse events reported after COVID-19 vaccination. Centers for Disease Control and Prevention, USA Available via https://www. cdc.gov/coronavirus/2019-ncov/vaccines/safety/adverse-events. $\mathrm{html}$

3. Engler RJ, Nelson MR, Collins LC Jr et al (2015) A prospective study of the incidence of myocarditis/pericarditis and new onset cardiac symptoms following smallpox and influenza vaccination. PLoS One. 10(3):e0118283

4. Montgomery J, Ryan M, Engler R et al (2021) Myocarditis following immunization with mRNA COVID-19 vaccines in members of the US military. JAMA Cardiol. https://doi.org/10.1001/ jamacardio.2021.2833
5. Catapano F, Marchitelli L, Cundari G et al (2021) Role of advanced imaging in COVID-19 cardiovascular complications. Insights Imaging. 24:12-28

6. Ferreira VM, Schulz-Menger J, Holmvang G et al (2018) Cardiovascular magnetic resonance in nonischemic myocardial inflammation: expert recommendations. J Am Coll Cardiol. 72: 3158-3176

7. Medicines and Healthcare products Regulatory Agency (MHRA, Government of the United Kingdom) (2021) Summary of yellow card reporting updated to include figures up to and including 17 nov. Available via https://www.gov.uk/government/publications/ coronavirus-covid-19-vaccine-adverse-reactions/coronavirusvaccine-summary-of-yellow-card-reporting

8. Mevorach D, Anis E, Cedar N et al (2021) Myocarditis after BNT162b2 mRNA vaccine against Covid-19 in Israel. N Eng1 J Med. 385:2140-2149

9. Public Health Agency of Canada (2021) Canadian COVID-19 vaccination safety report. November 26, 2021. Available via https:// health-infobase.canada.ca/covid-19/vaccine-safety/

10. Heymans S, Eriksson U, Lehtonen J, Cooper LT Jr (2016) The quest for new approaches in myocarditis and inflammatory cardiomyopathy. J Am Coll Cardiol. 68:2348-2364

11. Galea N, Marchitelli L, Pambianchi G et al (2021) T2-mapping increase is the prevalent imaging biomarker of myocardial involvement in active COVID-19: a cardiovascular magnetic resonance study. J Cardiovasc Magn Reson. 23(1):68

12. Pormohammad A, Zarei M, Ghorbani S et al (2021) Efficacy and safety of COVID-19 vaccines: a systematic review and metaanalysis of randomized clinical trials. Vaccines (Basel) 9:467

Publisher's note Springer Nature remains neutral with regard to jurisdictional claims in published maps and institutional affiliations.

\section{Affiliations}

\section{Emanuele Angelo Di Dedda ${ }^{1,2}$. Andrea Barison ${ }^{3} \cdot$ Giovanni Donato Aquaro $^{3} \cdot$ Tevfik F Ismail $^{4}$ • Alina Hua ${ }^{4}$. Cesare Mantini ${ }^{5}$. Fabrizio Ricci ${ }^{5}$. Gianluca Pontone ${ }^{6}$. Alessandra Volpe ${ }^{7,8}$ • Francesco Secchi ${ }^{9,10}$. Paolo Di Renzi ${ }^{11}$. Luigi Lovato $^{12} \cdot$ Fabio Niro $^{12}$. Carlo Liguori ${ }^{13} \cdot$ Chiara De Biase $^{14} \cdot$ Lorenzo Monti $^{2} \cdot$ Antonio Cirò $^{15}$. Riccardo Marano ${ }^{16,17}$. Luigi Natale $^{16,17}$. Eleonora Moliterno ${ }^{17}$. Antonio Esposito ${ }^{18,19}$. Davide Vignale ${ }^{18,19}$. Riccardo Faletti ${ }^{20}$ Marco Gatti $^{20}$ - Michele Porcu ${ }^{21}$ - Luca Saba ${ }^{21}$. Cristina Chimenti ${ }^{22}$ - Nicola Galea ${ }^{23}$. Marco Francone ${ }^{1,2}$ (D)}

Marco Francone

marco.francone@hunimed.eu

1 Department of Biomedical Sciences, Humanitas University, Via Rita Levi Montalcini 4, 20072 Pieve Emanuele, Milan, Italy

2 IRCCS Humanitas Research Hospital, via Manzoni 56, 20089 Rozzano, Milan, Italy

3 Department of Cardiology and Cardiovascular Medicine, Fondazione Toscana Gabriele Monasterio, Via Giuseppe Moruzzi, 1, 56124 Pisa, Italy

4 School of Biomedical Engineering and Imaging Sciences, King's College London, St Thomas' Hospital, Westminster Bridge Rd, London SE1 7EH, UK

5 Department of Neuroscience, Imaging and Clinical Sciences, "G.d'Annunzio" University of Chieti-Pescara, Via dei Vestini, 33, 66100 Chieti, CH, Italy

6 Department of Cardiovascular Imaging, Centro Cardiologico Monzino IRCCS, Via Carlo Parea, 4, 20138 Milan, MI, Italy

7 Division of Cardiology, Cardiovascular and Thoracic Department, Città della Salute e della Scienza, Corso Bramante, 88, 10126 Turin, TO, Italy

8 Cardiology, Department of Medical Sciences, University of Turin, Turin, Italy

9 Department of Biomedical Sciences for Health, Università Degli Studi Di Milano, Via Mangiagalli 31, 20133, Milan, Italy 
10 Unit of Radiology, IRCCS Policlinico San Donato, San Donato Milanese, Italy

11 Radiology Division, Ospedale "San Giovanni Calibita" Fatebenefratelli Isola Tiberina, Via di Ponte Quattro capi, 39, 00186 Rome, RM, Italy

12 Cardiovascular Radiology Unit - Radiology Department, AOU S. Orsola-Malpighi - IRCCS, Via Giuseppe Massarenti, 9, 40138 Bologna, BO, Italy

13 U.O.C. Diagnostica per Immagini. Ospedale del Mare - ASL NA1Centro, Via Enrico Russo, 80147 Naples, Italy

14 U.O.C. Cardiologia - UTIC- Emodinamica, Ospedale del Mare ASL NA1Centro, Via Enrico Russo, 80147 Naples, Italy

15 U.O.C. di Cardiologia e UTIC, Ospedale di Vimercate ASSTBrianza, Via Santi Cosma e Damiano, 10, 20871 Vimercate, Italy

16 Department of Radiological and Hematological Sciences, Section of Radiology, Università Cattolica del Sacro Cuore, L.go Francesco Vito 1, 00168 Rome, Italy
17 Department of Diagnostic Imaging, Oncological Radiotherapy and Hematology, Diagnostic Imaging Area, Fondazione Policlinico Universitario Agostino Gemelli, IRCCS, L.go Agostino Gemelli 8, 00168 Rome, Italy

18 Experimental Imaging Center, IRCCS Ospedale San Raffaele, Via Olgettina, 60, 20132 Milan, Italy

19 School of Medicine, Vita-Salute San Raffaele University, Milan, Italy

20 Radiology Unit, Department of Surgical Sciences, University of Turin, Città della Salute e della Scienza dì Torino, C.so Bramante 88, 10126 Turin, TO, Italy

21 Radiology Unit, Department of Medical Sciences, University of Cagliari, Via Università, 40, 09124 Cagliari, Italy

22 Department of Cardiovascular, Respiratory, Nephrologic, Anesthesiologic and Geriatric Sciences, "Sapienza" University of Rome, Viale Regina Elena 324, 00161 Rome, Italy

23 Department of Experimental Medicine, "Sapienza" University of Rome, Viale del Policlinico 155, 00161 Rome, Italy 